\title{
Uncovering the Key Role of Distortion in Tetrazine Ligations Guides the Design of Bioorthogonal Tools that Defy the Reactivity/Stability Tradeoff
}

\author{
Dennis Svatunek, ${ }^{\dagger, 1, *}$ Martin Wilkovitsch,${ }^{\dagger, \perp}$ Lea Hartmann, ${ }^{\dagger}$ Kendall N. Houk, ${ }^{\dagger}$ and Hannes Mikula ${ }^{\dagger}, *$ \\ ${ }^{\text {a }}$ Institute of Applied Synthetic Chemistry, TU Wien, 1060 Vienna, Austria \\ ${ }^{\mathrm{b}}$ Department of Chemistry and Biochemistry, University of California, Los Angeles, Los Angeles, 90095, United States
}

\begin{abstract}
The tetrazine/trans-cyclooctene ligation stands out from the bioorthogonal toolbox due to its exceptional reaction kinetics, enabling multiple molecular technologies in vitro and in living systems. Highly reactive 2-pyridyl-substituted tetrazines have become state-of-the-art for time-critical processes and selective reactions at very low concentration. It is widely accepted that the enhanced reactivity of these chemical tools is attributed to the electron-withdrawing effect of the heteroaryl substituent. In contrast, we show that observed reaction rates are way too high to be explained on this basis. Computational investigation of this phenomenon revealed that distortion of the tetrazine caused by intramolecular N-N repulsion plays a key role in accelerating the cycloaddition step. While we show that the limited stability of tetrazines under physiological conditions strongly correlates with the electron-withdrawing effect of the substituent, intramolecular destabilization increases the reactivity without reducing stability. Guided by these fundamental insights we demonstrate application in the design of highly reactive tetrazines with superior stability, finally evading the reactivity/stability trade-off for bioorthogonal tetrazine tools.
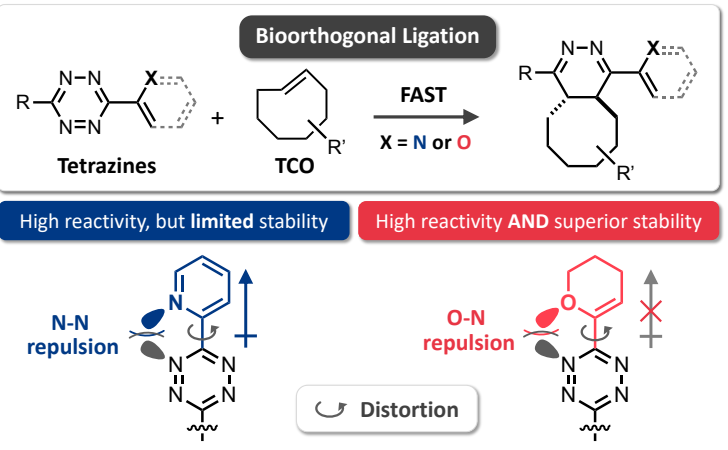

\section{INTRODUCTION}

The inverse electron demand Diels-Alder (IEDDA)initiated ligation of 1,2,4,5-tetrazines $(\mathrm{Tz})$ with strained dienophiles represents a group of exceptionally fast bioorthogonal reactions. ${ }^{[1-3]}$ In particular, trans-cyclooctene (TCO) derivatives $^{[4-6]}$ provide several orders of magnitude higher reactivity than other dienophiles such as cyclopropenes ${ }^{[7]}$ or norbornenes. ${ }^{[8]}$ In the rate-determining step the Tz first reacts with TCO in a [4+2]-cycloaddition to form a bicyclic intermediate that rapidly undergoes cycloreversion to give dihydropyridazines as ligation products (Fig. 1a). Due to high reaction rates, its biocompatibility, and versatility the Tz/TCO-ligation has found broad application in many fields, in particular enabling selective chemical reactions in living organisms. ${ }^{[2-3]}$ Very recently, bioorthogonal chemistry has entered Phase 1 clinical trials, with Tz/TCO-reactions currently being tested in humans, aiming for locally restricted prodrug activation to improve the selectivity of chemotherapeutics. ${ }^{[9-10]}$

In recent years, a variety of differently substituted tetrazines has been used for bioorthogonal reactions and in vivo chemistry, including bis-alkyl-substituted $\mathrm{Tz},{ }^{[11-15]}$ alkyl-aryl-Tz, ${ }^{[16-19]}$ mono-alkyl-Tz ${ }^{[20]}$ (alkyl-H-Tz) as well as highly reactive bisheteroaryl $^{[21-22]}$ and mono-aryl ${ }^{[23-26]}$ derivatives (aryl-H-Tz). These applications have motivated and fueled the development of advanced procedures for the synthesis of tetrazine scaffolds. ${ }^{[27-31]}$ In particular, 2-pyridyl-Tz are frequently used despite limited stability, because of their exceptionally high reactivity. This is commonly attributed to the electronwithdrawing effect of the 2-pyridyl substituent, resulting in a lowered orbital energy of the tetrazine, and thereby accelerating the IEDDA cycloaddition. ${ }^{[2-3,32]}$ Assuming that the reactivity is indeed controlled by frontier molecular orbital (FMO) interactions, we hypothesized that 4-pyridyl substituted Tz are even more reactive than their 2-pyridyl analogs. ${ }^{[33-35]}$

4-Pyridyl-Tz have previously been used for the design of fluorogenic probes. ${ }^{[36]}$ However, so far there is no comparative data on the IEDDA reaction kinetics of 2-pyridyl- and 4pyridyl-tetrazines. Therefore, we selected a series of phenyl (Ph), 2-pyridyl (2Pyr), 3-pyridyl (3Pyr), and 4-pyridyl (4Pyr) substituted Tz (Fig. 1b) to study the influence of the aryl substituent on the click reactivity in a combined experimental and computational approach, finally revealing the key role of $\mathrm{Tz}$ distortion in the reaction with TCO.

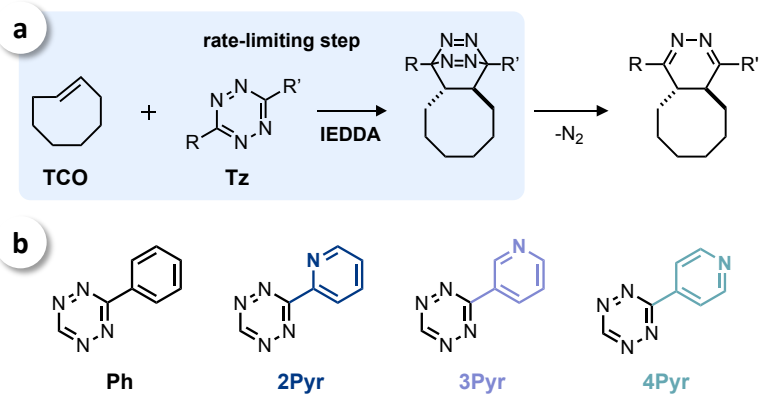

Figure 1. (a) Inverse electron demand Diels-Alder (IEDDA)-initiated tetrazine (Tz) ligation with trans-cyclooctene (TCO); (b) Selected Tz to investigate the impact of different aryl-substituents on click reactivity. 


\section{RESULTS AND DISCUSSION}

Theoretical calculations were performed using DFT at the $\omega \mathrm{B} 97 \mathrm{X}-\mathrm{D} / 6-311 \mathrm{G}(\mathrm{d}, \mathrm{p})-\mathrm{SMD}(1,4$-dioxane) level of theory, and orbital energies were calculated at the HF/6-311+G(d,p)SMD(1,4-dioxane)// $\omega$ B97X-D/6-311G(d,p) level of theory. A detailed description of the computational methods used within this study can be found in the Supporting Information. Energies for the reacting $\mathrm{LUMO}+1^{[32]}$ of tetrazines range from 1.29 to $0.89 \mathrm{eV}$. As expected, Ph shows the highest orbital energy followed by $\mathbf{2 P y r}$ and $\mathbf{3 P y r}$, which were calculated to have an almost equal LUMO+1 level, while 4Pyr shows the lowest orbital energy (Fig. 2a). According to FMO theory 4Pyr should thus indeed show an increased IEDDA reactivity compared to $\mathbf{2 P y r}$.

To compare these results with measured reaction rates we have prepared all selected mono-substituted aryl-tetrazines using a method recently published by Audebert and coworkers. ${ }^{[27]}$ Rate constants for the ligation with $\mathbf{T C O}{ }^{[37]}$ were measured by monitoring IEDDA reactions in anhydrous 1,4dioxane at $25^{\circ} \mathrm{C}$ by stopped-flow spectrophotometry. The measured rate constants span an order of magnitude ranging from $100 \mathrm{M}^{-1} \mathrm{~s}^{-1}$ for $\mathbf{P h}$, to $620 \mathrm{M}^{-1} \mathrm{~s}^{-1}$ for $\mathbf{2 P y r}$ (Fig. 2b). While the reactivity trend for $\mathbf{P h}, \mathbf{3 P y r}$, and $\mathbf{4 P y r}$ seems to be governed by FMO interactions (electron-withdrawing effects), $\mathbf{2 P y r}$ is significantly more reactive $(>3$-fold) than expected based on the respective orbital energy (Fig. 2c). Qualitatively equivalent results were obtained using calculated Kohn-Sham orbital energies (see Supporting Information, Fig. S1). Hence, the high IEDDA reactivity of $\mathbf{2 P y r}$ cannot be attributed to the electron-withdrawing effect of the 2-pyridyl substituent.

To investigate the origin of the observed reactivity trend for different pyridyl substituents a DFT study of the respective reactions was performed showing good correlation between the calculated free energies of activation $\left(\Delta \mathrm{G}^{\ddagger}\right)$ and the experimental values (Fig. S2). The calculated transition state geometries revealed a slight asynchronicity with very similar forming bond lengths across all four tetrazines. However, 2Pyr shows a slightly more synchronous bond formation (Fig. 3a).

For detailed investigation of the barrier heights we performed a distortion/interaction analysis (also referred to as activation/strain model ${ }^{[38]}$ on all four transition states (Fig. 3b) using the autoDIAS software package. ${ }^{[39]}$ This energy decomposition method was introduced independently by Houk and Bickelhaupt and has successfully been applied to investigate bioorthogonal cycloadditions. ${ }^{[32,40-42]}$ In this analysis, the energy of activation $\left(\Delta \mathrm{E}^{*}\right)$ is dissected into two parts, the a
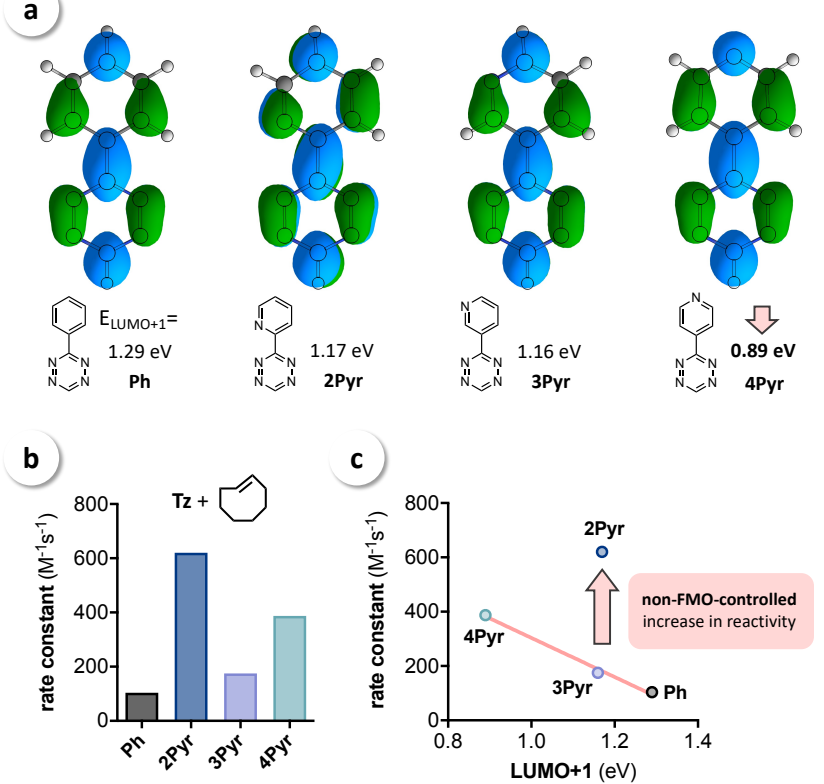

Figure 2. (a) LUMO+1 orbitals and orbital energies of mono-substituted $\mathrm{Tz}$ showing a significantly lower LUMO+1 energy for 4Pyr; (b) Rate constants for the reaction of $\mathbf{P h}, \mathbf{2 P y r}, 3 \mathbf{P y r}$, and $\mathbf{4 P y r}$ with $\mathbf{T C O}(1,4-$ dioxane, $25^{\circ} \mathrm{C}, \mathrm{n}=6, \mathrm{SD}<1 \%$ ); (c) Measured rate constants vs. calculated LUMO+1 energy.

distortion energy $\left(\Delta \mathrm{E}_{\mathrm{dist}}\right)$, which is needed to distort the isolated reactants into transition state geometry, and the interaction energy $\left(\Delta \mathrm{E}_{\mathrm{int}}\right)$ resulting from bringing the two distorted isolated fragments together. The analysis was performed at the transition state as a defined point for each reaction. Performing the distortion/interaction analysis at the transition state geometry can lead to skewed results if transition states at highly different forming bond lengths are compared. ${ }^{[43]}$ However, here the forming bond lengths are very similar in all cases, warranting the comparison at the transition state. To validate these results, an analysis at a consistent geometry with fixed bond lengths for all tetrazines was conducted and qualitatively provided the same results (see Supporting Information, Table S2). Interaction energies were calculated to be similar for all studied reactions (within $1.1 \mathrm{kcal} \mathrm{mol}^{-1}$ ) and do not explain the observed differences in IEDDA reactivity. However, $\Delta \mathrm{E}_{\text {dist }}$ for $\mathbf{2 P y r}$ is about $1.5 \mathrm{kcal} \mathrm{mol}^{-1}$ lower than for $\mathbf{P h}, \mathbf{3 P y r}$, and $\mathbf{4 P y r}$ demonstrating that the increased reactivity of $2 \mathbf{P y r}$ with TCO is mainly caused by a reduced distortion energy (Fig. 3b).

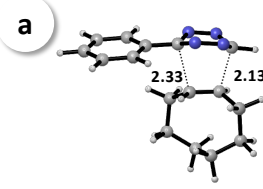

$\Delta \mathbf{G}^{\ddagger}=\quad 17.5$ $\mathrm{Ph}+\mathrm{TCO}$

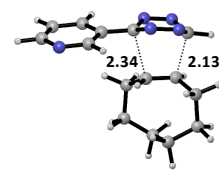

$\Delta \mathbf{G}^{\ddagger}=17.2$ $3 \mathrm{Pyr}+\mathrm{TCO}$

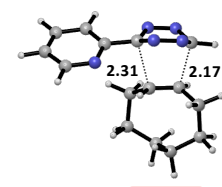

15.3

$2 \mathrm{Pyr}+\mathrm{TCO}$

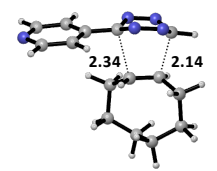

16.6

4 Pyr + TCO b

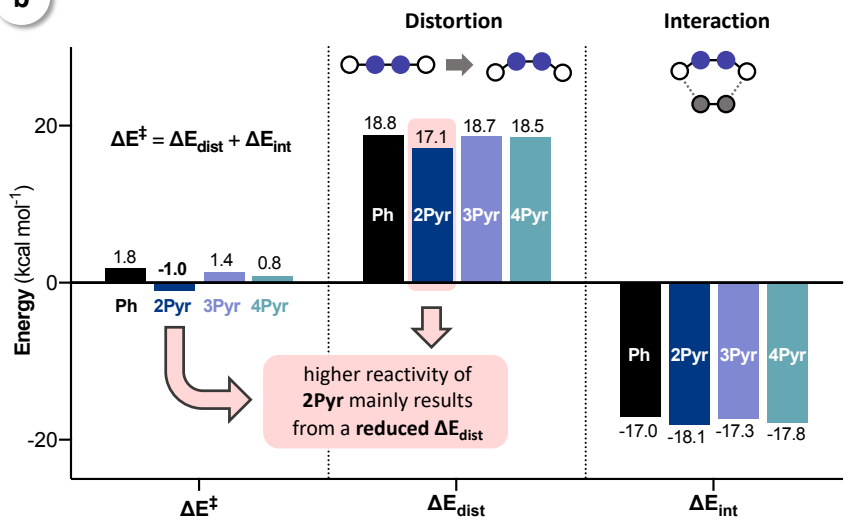

Figure 3. (a) Calculated transition state geometries and free energies of activation $\left(\Delta \mathrm{G}^{\ddagger}, \mathrm{kcal}^{\mathrm{mol}}{ }^{-1}\right)$ for the reaction of mono-substituted Tz (Ph, $\mathbf{2 P y r}, \mathbf{3 P y r}$, 4Pyr) and TCO; (b) Distortion/interaction analysis shows that the high reactivity of 2-pyridyl-substituted Tz results from a reduced distortion energy ( $\left.\Delta \mathrm{E}_{\mathrm{dist}}\right)$. 
To explain the lowered $\Delta \mathrm{E}_{\text {dist }}$ for aryl substituents with nitrogen atoms in 2-position we have focused on the geometries encountered at the transition state. In all cases the aryl moiety is tilted away from the allylic $\mathrm{CH}_{2}$ of TCO. For $\mathbf{P h}, \mathbf{3 P y r}$, and 4Pyr the dihedral angle is approximately $80^{\circ}$, which we reasoned is due to the steric demand of the allylic $\mathrm{CH}_{2}$. This hypothesis is in agreement with investigations of analogous reactions with ethylene (no allylic $\mathrm{CH}_{2}$ ), showing dihedral angles of approx. $90^{\circ}$ (Fig. 4a, Fig. S3). However, for 2Pyr we observed a much stronger tilt in the transition state for the reaction with TCO, with a dihedral angle of $63^{\circ}$, which did not change in the reaction with ethylene. These observations demonstrate that it is an intrinsic property of 2-pyridylsubstituted tetrazines rather than forced by steric interactions. In fact, the calculated geometry of $\mathbf{2 P y r}$ revealed that a nitrogen-nitrogen interaction destabilizes the reactant. This interaction becomes apparent when looking at the stabilization energies when going from an orthogonal (i.e., dihedral angle between the two aromatic rings of $90^{\circ}$ ) conformer in the reactant to the stable, coplanar conformer (Fig. 4b). For Ph, 3Pyr, and 4Pyr this stabilization energy based on conjugation between the ring systems is approximately $6 \mathrm{kcal} \mathrm{mol}^{-1}$. However, for 2Pyr the most stable conformation is not planar and the stabilization is only $3.5 \mathrm{kcal} \mathrm{mol}^{-1}$ due to a destabilizing interaction between the pyridyl nitrogen and the vicinal Tz nitrogen. This repulsion counteracts the stabilization due to conjugation of the aromatic systems, leading to an almost flat energy surface between $-30^{\circ}$ and $+30^{\circ}$ with a minimum at a dihedral angle of $12^{\circ}$ (Fig. 4b). At the transition state this $\mathrm{N}-\mathrm{N}$ repulsion can be avoided by rotation of the substituent, thereby increasing the distance between the interacting nitrogen atoms. In addition, the nitrogen lone pairs then point in different directions, further reducing the destabilizing interaction.
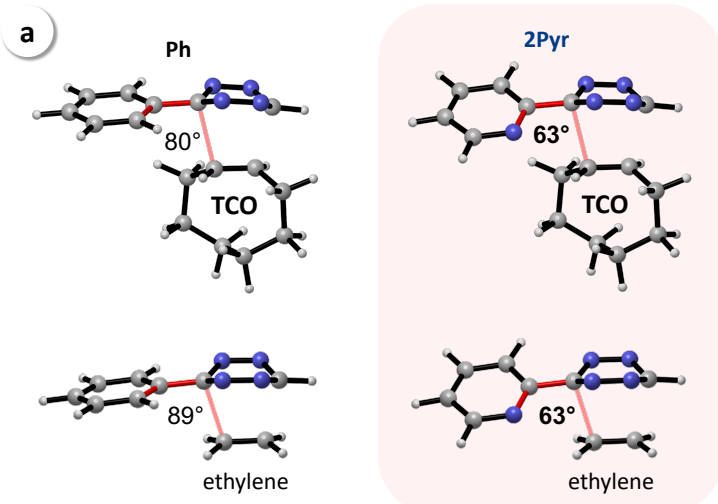

b

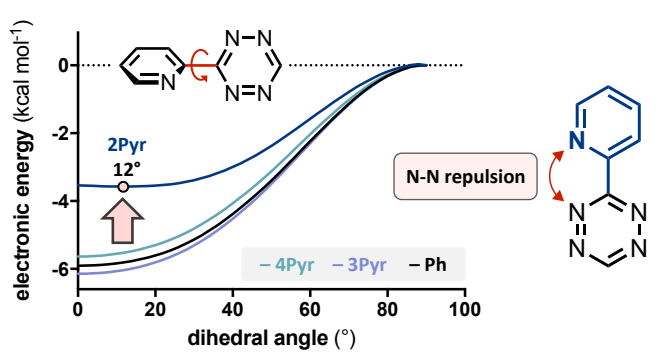

Figure 4. (a) Dihedral angle in the transition states for the reaction of $\mathbf{P h}$ and 2 Pyr with TCO and ethylene (for 3Pyr and 4Pyr see Fig. S3); (b) Calculated energy profiles for the rotation of the aryl-Tz bond showing that N-N repulsion reduces the stabilization energy for $\mathbf{2 P y r}$.
Hence, our results revealed that the high reactivity of 2pyridyl-tetrazines cannot be explained by the electronwithdrawing nature of the heteroaryl-substituent only, but moreover by intramolecular N-N repulsion, finally uncovering the mechanistic key role of distortion in tetrazine ligations.

To confirm that these findings can be translated to physiological conditions, additional IEDDA reactions were carried out in Dulbecco's phosphate buffered saline (DPBS) at $37^{\circ} \mathrm{C}$. Due to the limited stability of the mono-substituted $\mathrm{H}$ tetrazines in aqueous solution we have prepared the respective methyl-Tz MePh, Me2Pyr, Me3Pyr, and Me4Pyr, and determined the second order rate constants for the reaction with water-soluble TCO-PEG $\mathbf{T H}_{\mathbf{4}}$ (Fig. 5a). This TCO derivative has been prepared starting from axially configured TCO-OH, a frequently used tag for the design of in vivo chemical tools. ${ }^{[2,44-45]}$ The observed relative reactivity profile almost exactly matches the data as previously obtained for the reactions of the corresponding aryl-H-Tz with TCO in 1,4-dioxane (cf. Fig. 2b), with Me2Pyr showing the highest rate constant in the reaction with $\mathbf{T C O}-\mathbf{P E G}_{4}$ (Fig. 5a).

It is generally accepted that increasing the reactivity of the tetrazine by using electron-withdrawing substituents leads to reduced stability in aqueous/biological media due to accelerated attack of nucleophiles causing Tz degradation (Fig. 5b). ${ }^{[1-}$ 3,46-47] However, when working with buffered aqueous solutions of the aryl-methyl-Tz we noticed a significantly faster degradation of Me4Pyr compared to Me2Pyr (as indicated by accelerated fading of the characteristic pink color of $\mathrm{Tz}$ ), despite the higher reactivity of the 2-pyridyl-Tz. To investigate this observation, MePh, Me2Pyr, Me3Pyr, and Me4Pyr were incubated in full cell growth medium (Dulbecco's Modified Eagle's medium, DMEM, incl. 10\% fetal bovine serum, FBS)

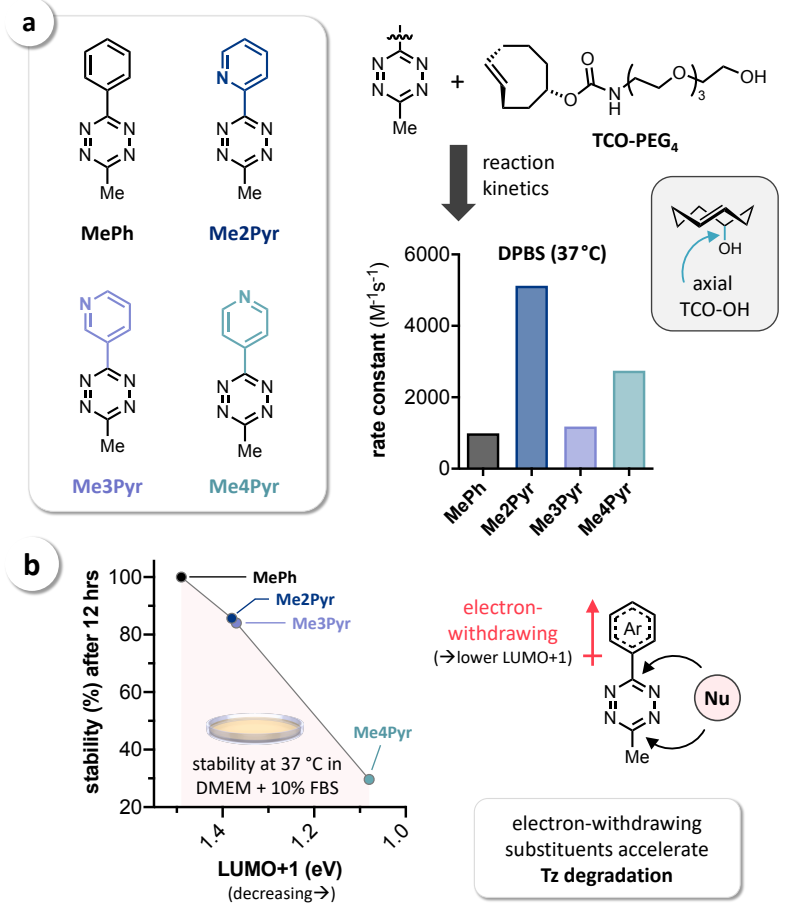

Figure 5. (a) Reaction kinetics of MePh, Me2Pyr, Me3Pyr and Me4Pyr in buffered aqueous solution (DPBS) at $37{ }^{\circ} \mathrm{C}$ using water-soluble TCO$\mathbf{P E G}_{4}(\mathrm{n}=6, \mathrm{SD}<1 \%)$; (b) Stability of $\mathrm{Tz}$ in cell growth medium at $37^{\circ} \mathrm{C}(\mathrm{n}=3, \mathrm{SD}<5 \%)$ revealing accelerated degradation with decreasing LUMO+1 energy as a measure for increased electron-withdrawing of the (hetero)aryl substituent, promoting the attack of nucleophiles $(\mathrm{Nu})$. 
at $37^{\circ} \mathrm{C}$ and $\mathrm{Tz}$ stability was monitored by spectrophotometry (Fig. 5b). Indeed, Me4Pyr degraded much faster (30\% intact after 12 hours) compared to Me3Pyr and Me2Pyr (approx. $85 \%$ ), and MePh (>95\%). These results correlate well with decreasing LUMO+1 energy ${ }^{[32]}$ of the Tz (Fig. 5b), which we used as a measure for increased electron-withdrawing of the (hetero)aryl substituent. This finding is in agreement with the hypothesis of accelerated nucleophilic attack leading to $\mathrm{Tz}$ degradation. Me2Pyr is thus not only more reactive than predicted by FMO theory, but moreover shows a significantly higher stability than expected based on its IEDDA reactivity.

Motivated by these key mechanistic insights, we aimed to exploit intramolecular $\mathrm{Tz}$ interactions to evade the reactivity/stability trade-off, enabling the design of advanced bioorthogonal tetrazine tools. Very recently, Joseph Fox and coworkers have reported on the increased IEDDA reactivity of vinyl ether-substituted Tz. ${ }^{[29]}$ Inspired by these results, we hypothesized that the observed unexpected boost in reactivity is due to lowered distortion energies, caused by intramolecular O-N-repulsion (Fig. 6a). Considering the non-electronwithdrawing character of the vinyl ether-moiety, such scaffolds would thus potentially enable the development of highly reactive tetrazines with substantially increased stability. Computational investigations were thus performed using the $\mathrm{Tz}$ structures MVE (methylvinyl ether-Tz) and MV (methylvinyl$\mathrm{Tz}$ ). The optimized geometries revealed that an oxygennitrogen interaction destabilizes MVE leading to a reduced rotational barrier of $3.9 \mathrm{kcal} \mathrm{mol}^{-1}$ for MVE (similar to 2Pyr) in comparison to $5.1 \mathrm{kcal} \mathrm{mol}^{-1}$ for $\mathbf{M V}$ (Fig. 6b). The calculated $\mathrm{LUMO}+1$ energies moreover indicate a non-electronwithdrawing character of both substituents $(\sim 1.5 \mathrm{eV}$ in contrast to $0.89 \mathrm{eV}$ as calculated for $\mathbf{4 P y r}$ ). The transition state geometries for the reaction with TCO (Fig. 6c) showed a significantly stronger tilt of the vinyl-Tz bond in the case of the vinyl ether-Tz MVE (dihedral angle of $66^{\circ}$ ). The calculated distortion energies $\left(\Delta \mathrm{E}_{\text {dist }}\right)$ finally confirmed $\mathrm{O}-\mathrm{N}$ repulsion to play a crucial role regarding the potentially increased reactivity of MVE, as indicated by a calculated free energy of activation $\left(\Delta \mathrm{G}^{*}\right)$ of $16.0 \mathrm{kcal} / \mathrm{mol}$.
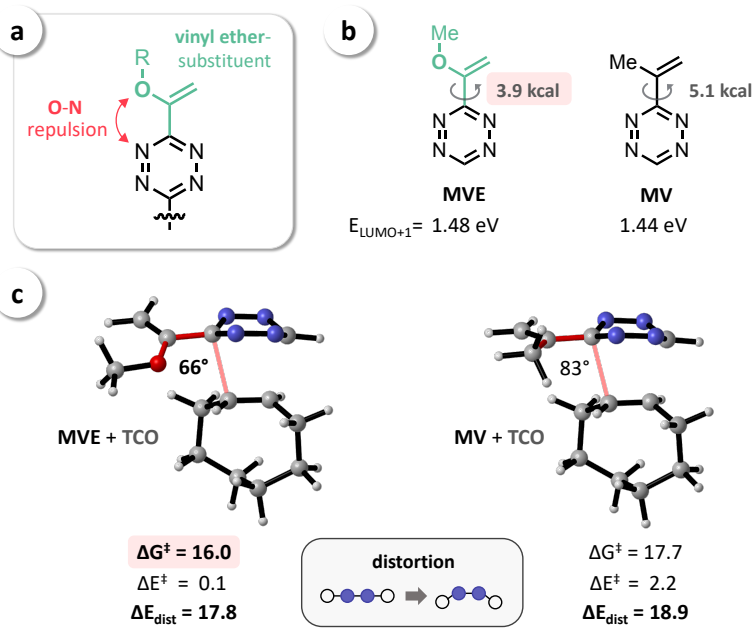

$\Delta \mathrm{G}^{\ddagger}=17.7$

$\Delta \mathrm{E}^{\ddagger}=2.2$

$\Delta \mathrm{E}_{\mathrm{dist}}=18.9$

Figure 6. (a) O-N interaction destabilizes and thereby increases the reactivity of vinyl ether-Tz; (b) Computational analysis revealed a reduced rotational barrier for $\mathbf{M V E}$ in comparison to $\mathbf{M V}$, and a relatively high LUMO+1 energy of $1.46 \mathrm{eV}$; (c) Optimized transition state geometries $(\mathrm{Tz}+\mathrm{TCO})$ and distortion/interaction analysis confirmed $\mathrm{O}-\mathrm{N}$ repulsion to be the main reason for the increased reactivity of MVE, as indicated by the calculated values for $\Delta \mathrm{G}^{\ddagger}, \Delta \mathrm{E}^{\dot{\hbar}}$ and $\Delta \mathrm{E}_{\text {dist }}\left(\mathrm{kcal} \mathrm{mol}^{-1}\right)$.
Encouraged by the computational results, we have prepared the vinyl ether-Tz MeEVE and the 3,4-dihydro- $2 H$ pyran (DHP)-substituted tetrazines MeDHP and DHP (Fig. $7 \mathrm{a}$; for details on synthetic procedures see the Supporting Information). Second order rate constants for the reactions of these Tz with TCO-PEG 4 in DPBS at $37^{\circ} \mathrm{C}$ were determined by stopped-flow spectrophotometry. The IEDDA reactivity of MeEVE $\left(2750 \mathrm{M}^{-1} \mathrm{~s}^{-1}\right)$ was shown to match the value measured for Me4Pyr (2740 $\left.\mathrm{M}^{-1} \mathrm{~s}^{-1}\right)$. In comparison, the cyclic vinyl ether-Tz MeDHP was observed to be less reactive $\left(1820 \mathrm{M}^{-1} \mathrm{~s}^{-1}\right)$, though still significantly faster than the aryl-Tz MePh $\left(990 \mathrm{M}^{-1} \mathrm{~s}^{-1}\right)$. As expected, we observed a high reactivity of the bis-vinyl ether-Tz $\mathbf{D H} \mathbf{P}_{2}\left(6450 \mathrm{M}^{-1} \mathrm{~s}^{-1}\right)$, exceeding the rate constant of Me2Pyr $\left(5120 \mathrm{M}^{-1} \mathrm{~s}^{-1}\right)$ by approx. 25\% (Fig. $7 \mathrm{a}$. These results finally confirm the distortion-induced IEDDA acceleration due to intramolecular O-N repulsion and show that increased reactivities similar to pyridyl-Tz can be achieved by using non-electron-withdrawing vinyl ether substituents
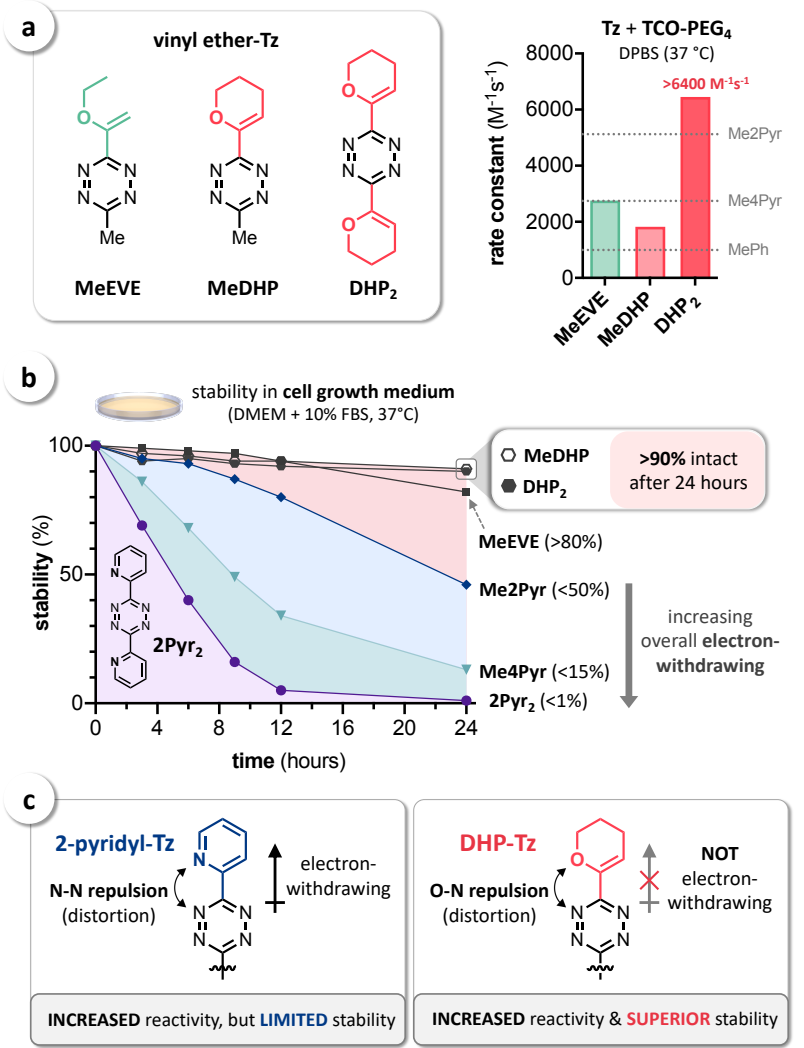

Figure 7. (a) Vinyl ether-Tz MeEVE, MeDHP and $\mathbf{D H P}_{2}$, and second order rate constants for the reaction with TCO-PEG $_{4}$ in buffered aqueous solution (DPBS) at $37^{\circ} \mathrm{C}(\mathrm{n}=6, \mathrm{SD}<1 \%)$; (b) Stability of pyridyl- and vinyl ether-Tz under physiological conditions (full cell growth medium, $37^{\circ} \mathrm{C}, \mathrm{n}=3, \mathrm{SD}<5 \%$ ) revealing accelerated degradation of pyridyl-Tz in contrast to the exceptional stability of DHP-substituted Tz; (c) Destabilizing 2-pyridyl-substituents increase the IEDDA reactivity of Tz, also leading to limited stability. In contrast, destabilizing, but non-electronwithdrawing DHP enables the design of tetrazines with high reactivity and superior stability.

Subsequent investigation of $\mathrm{Tz}$ stability in full cell growth medium at $37{ }^{\circ} \mathrm{C}$ moreover revealed a high stability of vinyl ether-Tz, in particular of MeDHP and $\mathbf{D H P} \mathbf{P}_{2}$, in contrast to the limited stability of pyridyl-substituted Tz (Fig. 7b). For instance, despite being equal in reactivity, MeDHP is signifi- 
cantly more stable than 4 Pyr $(>90 \%$ vs. $<15 \%)$. Notably, DHP-substituents do not lead to decreased stability, as shown by the data obtained for symmetrical $\mathbf{D H} \mathbf{P}_{2}$ and bis(2pyridyl)tetrazine $\left(\mathbf{2 P y r}_{\mathbf{2}}\right)$. While installation of a second DHP had no detrimental effect on stability ( $>90 \%$ for both MeDHP and $\mathbf{D H} \mathbf{P}_{2}$ ), an additional 2-pyridyl substituent resulted in almost complete $\mathrm{Tz}$ degradation within 24 hours. Despite showing very fast IEDDA reaction with TCO-PEG $_{4}$ $\left(69,400 \mathbf{M}^{-1} \mathrm{~s}^{-1}\right)$, only $<1 \%$ of intact $\mathbf{2} \mathbf{P y r}_{2}$ was detected at the end of the experiment, in comparison to $>90 \%$ of $\mathbf{D H} \mathbf{P}_{2}$ (Fig. 7b). In applications that require extended stability of the $\mathrm{Tz}$ $(>10 \mathrm{~h}), \mathbf{D H P} \mathbf{P}_{2}$ thus outperforms even highly reactive $\mathbf{2} \mathbf{P y r}_{\mathbf{2}}$ (Fig. S4). Overall, these results show that non-electronwithdrawing DHP-substituents can be used to significantly increase the IEDDA reactivity of tetrazines, while also drastically improving compound stability (Fig. 7c).

\section{CONCLUSIONS}

Our detailed investigation of the reactivity of pyridyl-Tz uncovered the key role of reduced $\mathrm{Tz}$ distortion energies caused by destabilizing intramolecular interactions. Guided by these insights we have been able to show that vinyl ethersubstituents increase IEDDA reactivity without accelerating $\mathrm{Tz}$ degradation under physiological conditions. In particular, 3,4-dihydro- $2 H$-pyran (DHP) was identified as a destabilizing, non-electron-withdrawing substituent, enabling the design of tetrazines with high reactivity and superior stability. Finally evading the reactivity/stability trade-off, these findings will thus be instrumental in the development of next-generation bioorthogonal tools, in particular for in vivo chemical strategies that require long-term tetrazine stability. ${ }^{[9,48]}$

\section{ASSOCIATED CONTENT}

\section{Supporting Information}

Computational methods, organic synthesis, reaction kinetics, compound stability and characterization, copies of NMR spectra (PDF)

Coordinates of all calculated geometries (xyz)

\section{AUTHOR INFORMATION}

\section{Corresponding Author}

* hannes.mikula@tuwien.ac.at; orcid.org/0000-0002-9218-9722

* dennis.svatunek@tuwien.ac.at; orcid.org/0000-0003-1101-2376

\section{Author Contributions}

The manuscript was written through contributions of all authors. All authors have given approval to the final version of the manuscript.

${ }^{\perp}$ D.S. and M.W. contributed equally.

\section{Funding Sources}

This project has received funding from the European Union's EU Framework Programme for Research and Innovation Horizon 2020, under grant agreement no. 668532.

\section{Notes}

The authors declare no competing financial interest.

\section{ACKNOWLEDGMENT}

This project has received funding from the European Union's EU Framework Programme for Research and Innovation Horizon 2020, under grant agreement no. 668532. The authors thank Jesper L. Kristensen (University of Copenhagen) for the helpful discussion. The computational results presented have been achieved using the Vienna Scientific cluster (VSC) at TU Wien and the Hoffman2 cluster at UCLA. D.S. is grateful to the Theodor Körner fund (Vienna, Austria) for financial support.

\section{REFERENCES}

[1] Nguyen, S. S.; Prescher, J. A. Developing bioorthogonal probes to span a spectrum of reactivities. Nat. Rev. Chem. 2020, 4, 476-489.

[2] Mayer, S.; Lang, K. Tetrazines in Inverse-Electron-Demand Diels-Alder Cycloadditions and Their Use in Biology. Synthesis 2017, 49, 830-848.

[3] Oliveira, B. L.; Guo, Z.; Bernardes, G. J. L. Inverse electron demand Diels-Alder reactions in chemical biology. Chem. Soc. Rev. 2017, 46, 4895-4950.

[4] Selvaraj, R.; Fox, J. M. trans-Cyclooctene - a stable, voracious dienophile for bioorthogonal labeling. Curr. Opin. Chem. Biol. 2013, 17, 753-760.

[5] Darko, A.; Wallace, S.; Dmitrenko, O.; Machovina, M. M.; Mehl, R. A.; Chin, J. W.; Fox, J. M. Conformationally Strained transCyclooctene with Improved Stability and Excellent Reactivity in Tetrazine Ligation. Chem Sci 2014, 5, 3770-3776.

[6] Pigga, J. E.; Rosenberger, J. E.; Jemas, A.; Boyd, S. J.; Dmitrenko, O.; Xie, Y.; Fox, J. M. General, Divergent Platform for Diastereoselective Synthesis of trans-Cyclooctenes with High Reactivity and Favorable Physiochemical Properties. Angew. Chem. Int. Ed. 2021, 60, 14975-14980.

[7] Yang, J.; Šečkute, J.; Cole, C. M.; Devaraj, N. K. Live-Cell Imaging of Cyclopropene Tags with Fluorogenic Tetrazine Cycloadditions. Angew. Chem. Int. Ed. 2012, 51, 7476-7479.

[8] Knall, A.-C.; Hollauf, M.; Slugovc, C. Kinetic studies of inverse electron demand Diels-Alder reactions (iEDDA) of norbornenes and 3,6-dipyridin-2-yl-1,2,4,5-tetrazine. Tetrahedron Lett. 2014, $55,4763-4766$

[9] Wu, K.; Yee, N. A.; Srinivasan, S.; Mahmoodi, A.; Zakharian, M.; Mejia Oneto, J. M.; Royzen, M. Click activated protodrugs against cancer increase the therapeutic potential of chemotherapy through local capture and activation. Chem. Sci. 2021, 12, 1259-1271.

[10] Srinivasan, S.; Yee, N. A.; Wu, K.; Zakharian, M.; Mahmoodi, A.; Royzen, M.; Mejía Oneto, J. M. SQ3370 Activates Cytotoxic Drug via Click Chemistry at Tumor and Elicits Sustained Responses in Injected and Non-Injected Lesions. Adv. Ther. 2021, 4, 2000243.

[11] Denk, C.; Svatunek, D.; Filip, T.; Wanek, T.; Lumpi, D.; Fröhlich, J.; Kuntner, C.; Mikula, H. Development of a18F-Labeled Tetrazine with Favorable Pharmacokinetics for Bioorthogonal PET Imaging. Angew. Chem. Int. Ed. 2014, 53, 9655-9659.

[12] Denk, C.; Svatunek, D.; Mairinger, S.; Stanek, J.; Filip, T.; Matscheko, D.; Kuntner, C.; Wanek, T.; Mikula, H. Design, Synthesis, and Evaluation of a Low-Molecular-Weight 11C-Labeled Tetrazine for Pretargeted PET Imaging Applying Bioorthogonal in Vivo Click Chemistry. Bioconjugate Chem. 2016, 27, 1707-1712.

[13] Versteegen, R. M.; Rossin, R.; ten Hoeve, W.; Janssen, H. M.; Robillard, M. S. Click to Release: Instantaneous Doxorubicin Elimination upon Tetrazine Ligation. Angew. Chem. Int. Ed. 2013, 52, 14112-14116

[14] Carlson, J. C. T.; Mikula, H.; Weissleder, R. Unraveling Tetrazine-Triggered Bioorthogonal Elimination Enables Chemical Tools for Ultrafast Release and Universal Cleavage. J. Am. Chem. Soc. 2018, 140, 3603-3612.

[15] Rossin, R.; Versteegen, R. M.; Wu, J.; Khasanov, A.; Wessels, H. J.; Steenbergen, E. J.; ten Hoeve, W.; Janssen, H. M.; van Onzen, A. H. A. M.; Hudson, P. J.; Robillard, M. S. Chemically triggered drug release from an antibody-drug conjugate leads to potent antitumour activity in mice. Nat. Commun. 2018, 9, 1484. 
[16] Fan, X.; Ge, Y.; Lin, F.; Yang, Y.; Zhang, G.; Ngai, W. S. C.; Lin, Z.; Zheng, S.; Wang, J.; Zhao, J.; Li, J.; Chen, P. R. Optimized Tetrazine Derivatives for Rapid Bioorthogonal Decaging in Living Cells. Angew. Chem. Int. Ed. 2016, 55, 14046-14050.

[17] Werther, P.; Yserentant, K.; Braun, F.; Kaltwasser, N.; Popp, C.; Baalmann, M.; Herten, D.-P.; Wombacher, R. Live-Cell Localization Microscopy with a Fluorogenic and Self-Blinking Tetrazine Probe. Angew. Chem. Int. Ed. 2020, 59, 804-810.

[18] Tu, J.; Svatunek, D.; Parvez, S.; Liu, A. C.; Levandowski, B. J.; Eckvahl, H. J.; Peterson, R. T.; Houk, K. N.; Franzini, R. M. Stable, Reactive, and Orthogonal Tetrazines: Dispersion Forces Promote the Cycloaddition with Isonitriles. Angew. Chem. Int. Ed. 2019, 58, 9043-9048.

[19] Sarris, A. J. C.; Hansen, T.; de Geus, M. A. R.; Maurits, E.; Doelman, W.; Overkleeft, H. S.; Codée, J. D. C.; Filippov, D. V.; van Kasteren, S. I. Fast and pH-Independent Elimination of transCyclooctene by Using Aminoethyl-Functionalized Tetrazines. Chem. Eur. J. 2018, 24, 18075-18081.

[20] Wilkovitsch, M.; Haider, M.; Sohr, B.; Herrmann, B.; Klubnick, J.; Weissleder, R.; Carlson, J. C. T.; Mikula, H. A Cleavable C2Symmetric trans-Cyclooctene Enables Fast and Complete Bioorthogonal Disassembly of Molecular Probes. J. Am. Chem. Soc. 2020, 142, 19132-19141.

[21] Rossin, R.; Renart Verkerk, P.; van den Bosch, S. M.; Vulders, R. C. M.; Verel, I.; Lub, J.; Robillard, M. S. In Vivo Chemistry for Pretargeted Tumor Imaging in Live Mice. Angew. Chem. Int. Ed. 2010, 49, 3375-3378.

[22] Stéen, E. J. L.; Jørgensen, J. T.; Denk, C.; Battisti, U. M.; Nørregaard, K.; Edem, P. E.; Bratteby, K.; Shalgunov, V.; Wilkovitsch, M.; Svatunek, D.; Poulie, C. B. M.; Hvass, L.; Simón, M.; Wanek, T.; Rossin, R.; Robillard, M.; Kristensen, J. L.; Mikula, H.; Kjaer, A.; Herth, M. M. Lipophilicity and Click Reactivity Determine the Performance of Bioorthogonal Tetrazine Tools in Pretargeted In Vivo Chemistry. ACS Pharmacol. Transl. Sci. 2021, 4, 824 833.

[23] Meimetis, L. G.; Carlson, J. C. T.; Giedt, R. J.; Kohler, R. H.; Weissleder, R. Ultrafluorogenic Coumarin-Tetrazine Probes for RealTime Biological Imaging. Angew. Chem. Int. Ed. 2014, 53, 75317534.

[24] Zeglis, B. M.; Sevak, K. K.; Reiner, T.; Mohindra, P.; Carlin, S. D.; Zanzonico, P.; Weissleder, R.; Lewis, J. S. A Pretargeted PET Imaging Strategy Based on Bioorthogonal Diels-Alder Click Chemistry. J. Nucl. Med. 2013, 54, 1389-1396.

[25] Denk, C.; Wilkovitsch, M.; Aneheim, E.; Herth, M. M.; Jensen, H.; Lindegren, S.; Mikula, H. Multifunctional Clickable Reagents for Rapid Bioorthogonal Astatination and Radio-Crosslinking. ChemPlusChem 2019, 84, 775-778.

[26] Garcia-Vazquez, R.; Battisti, U. M.; Jørgensen, J. T.; Shalgunov, V.; Hvass, L.; Stares, D. L.; Nymann Petersen, I.; Crestey, F.; Löffler, A.; Svatunek, D.; Kristensen, J. L.; Mikula, H.; Kjaer, A.; Herth, M. M. Direct Cu-mediated aromatic 18F-labeling of highly reactive tetrazines for pretargeted bioorthogonal PET imaging. Chem. Sci. 2021, 12, 11668-11675.

[27] Qu, Y.; Sauvage, F.-X.; Clavier, G.; Miomandre, F.; Audebert, P. Metal-Free Synthetic Approach to 3-Monosubstituted Unsymmetrical 1,2,4,5-Tetrazines Useful for Bioorthogonal Reactions. Angew. Chem. Int. Ed. 2018, 57, 12057-12061.

[28] Yang, J.; Karver, M. R.; Li, W.; Sahu, S.; Devaraj, N. K. Metal-Catalyzed One-Pot Synthesis of Tetrazines Directly from Aliphatic Nitriles and Hydrazine. Angew. Chem. Int. Ed. 2012, 51, 5222-5225.

[29] Xie, Y.; Fang, Y.; Huang, Z.; Tallon, A. M.; am Ende, C. W.; Fox, J. M. Divergent Synthesis of Monosubstituted and Unsymmetrical 3,6-Disubstituted Tetrazines from Carboxylic Ester Precursors. Angew. Chem. Int. Ed. 2020, 59, 16967-16973.

[30] Mao, W.; Shi, W.; Li, J.; Su, D.; Wang, X.; Zhang, L.; Pan, L.; Wu, X.; Wu, H. Organocatalytic and Scalable Syntheses of Unsymmetrical 1,2,4,5-Tetrazines by Thiol-Containing Promotors. Angew. Chem. Int. Ed. 2019, 58, 1106-1109.
[31] Lambert, W. D.; Fang, Y.; Mahapatra, S.; Huang, Z.; am Ende, C. W.; Fox, J. M. Installation of Minimal Tetrazines through Silver-Mediated Liebeskind-Srogl Coupling with Arylboronic Acids. J. Am. Chem. Soc. 2019, 141, 17068-17074.

[32] Liu, F.; Liang, Y.; Houk, K. N. Theoretical Elucidation of the Origins of Substituent and Strain Effects on the Rates of Diels-Alder Reactions of 1,2,4,5-Tetrazines. J. Am. Chem. Soc. 2014, 136, 1148311493.

[33] Shin, Y.-H.; Koh, H.-J.; Um, I.-H. Kinetic Study on Alkaline Hydrolysis of 2-Pyridyl and 4-Pyridyl X-substituted-Benzoates: Effects of Benzoyl Substituent X and Leaving-Group Basicity on Reactivity and Reaction Mechanism. Bull. Korean Chem. Soc. 2017, 38, 1138-1142.

[34] Hansch, C.; Leo, A.; Taft, R. W. A survey of Hammett substituent constants and resonance and field parameters. Chem. Rev. 1991, 91, 165-195.

[35] Eicher, T.; Hauptmann, S.; Speicher, A., Six-Membered Heterocycles: Sections 6.10-6.14. In The Chemistry of Heterocycles, 2nd ed.; Eicher, T.; Hauptmann, S.; Speicher, A., Eds. Wiley-VCH: Weinheim, Germany, 2003; pp 257-310.

[36] Vázquez, A.; Dzijak, R.; Dračínský, M.; Rampmaier, R.; Siegl, S. J.; Vrabel, M. Mechanism-Based Fluorogenic trans

-Cyclooctene-Tetrazine Cycloaddition. Angew. Chem. Int. Ed 2017, 56, 1334-1337.

[37] Svatunek, D.; Denk, C.; Rosecker, V.; Sohr, B.; Hametner, C.; Allmaier, G.; Frohlich, J.; Mikula, H. Efficient low-cost preparation of trans-cyclooctenes using a simplified flow setup for photoisomerization. Monatsh. Chem. 2016, 147, 579-585.

[38] Bickelhaupt, F. M.; Houk, K. N. Analyzing Reaction Rates with the Distortion/Interaction-Activation Strain Model. Angew. Chem. Int. Ed. 2017, 56, 10070-10086.

[39] Svatunek, D.; Houk, K. N. autoDIAS: a python tool for an automated distortion/interaction activation strain analysis. J. Comput. Chem. 2019, 40, 2509-2515.

[40] Svatunek, D.; Houszka, N.; Hamlin, T. A.; Bickelhaupt, F. M.; Mikula, H. Chemoselectivity of Tertiary Azides in Strain-Promoted Alkyne-Azide Cycloadditions. Chem. Eur. J. 2019, 25, 754-758.

[41] Gold, B.; Aronoff, M. R.; Raines, R. T. Decreasing Distortion Energies without Strain: Diazo-Selective 1,3-Dipolar Cycloadditions. J. Org. Chem. 2016, 81, 5998-6006.

[42] Liang, Y.; Mackey, J. L.; Lopez, S. A.; Liu, F.; Houk, K. N. Control and Design of Mutual Orthogonality in Bioorthogonal $\mathrm{Cy}-$ cloadditions. J. Am. Chem. Soc. 2012, 134, 17904-17907.

[43] Hamlin, T. A.; Svatunek, D.; Yu, S.; Ridder, L.; Infante, I.; Visscher, L.; Bickelhaupt, F. M. Elucidating the Trends in Reactivity of Aza-1,3-Dipolar Cycloadditions. Eur. J. Org. Chem. 2019, 2019, 378-386.

[44] Rossin, R.; van Duijnhoven, S. M. J.; Läppchen, T.; van den Bosch, S. M.; Robillard, M. S. Trans-Cyclooctene Tag with Improved Properties for Tumor Pretargeting with the Diels-Alder Reaction. Mol. Pharm. 2014, 11, 3090-3096.

[45] Rossin, R.; van den Bosch, S. M.; ten Hoeve, W.; Carvelli, M.; Versteegen, R. M.; Lub, J.; Robillard, M. S. Highly Reactive trans-Cyclooctene Tags with Improved Stability for Diels-Alder Chemistry in Living Systems. Bioconjugate Chem. 2013, 24, 12101217.

[46] Tolshchina, S. G.; Rusinov, G. L.; Charushin, V. N. 1,2,4,5Tetrazines and Azolo[1,2,4,5]tetrazines: Synthesis and Reactions with Nucleophiles. Chemistry of Heterocyclic Compounds 2013, 49, 66-91.

[47] Karver, M. R.; Weissleder, R.; Hilderbrand, S. A. Synthesis and Evaluation of a Series of 1,2,4,5-Tetrazines for Bioorthogonal Conjugation. Bioconjugate Chem. 2011, 22, 2263-2270.

[48] van Onzen, A. H. A. M.; Versteegen, R. M.; Hoeben, F. J. M.; Filot, I. A. W.; Rossin, R.; Zhu, T.; Wu, J.; Hudson, P. J.; Janssen, H. M.; ten Hoeve, W.; Robillard, M. S. Bioorthogonal Tetrazine Carbamate Cleavage by Highly Reactive trans-Cyclooctene. J. Am. Chem. Soc. 2020, 142, 10955-10963. 